\title{
Dynamical Stability and Pure Thermodynamic Phases
}

\author{
Heidi Narnhofer \\ Institut für Theoretische Physik der Universität Wien, Wien, Austria \\ Derek W. Robinson \\ Departement de Physique, Universite d'Aix-Marseille II, Luminy, France
}

Received July 15, 1974

\begin{abstract}
A notion of stability of dynamics under distant perturbations is introduced. It is demonstrated, for quasi-local systems, that the stability of an equilibrium state under the same perturbations implies the state is factorial, i.e. strongly clustering in space. We also characterize the set of perturbations necessary to ensure the equivalence of stability and factorialness.
\end{abstract}

\section{Introduction}

Several characterizations of pure thermodynamic phases have been proposed; each such proposal emphasizes a different aspect of the equilibrium phenomena. The aspects which have received most attention are either kinematical

a) spatial cluster properties,

b) extremality among the class of all equilibrium states, or dynamical

c) stability under dynamical perturbations,

d) temporal cluster properties.

The purpose of this note is to examine some of the interrelationships between properties $a, b$, and $c$, in the framework of quantum statistical mechanics.

For simple quantum models, such as spin systems with short range interactions, the connections between points a and $b$, have been reasonably well understood. The dynamics in these models is provided by a strongly continuous one-parameter group of automorphisms $\tau$ of a $C^{*}$ algebra $\mathfrak{A}$ of kinematical observables and the thermodynamic limits of finite volume Gibbs equilibrium states form the set (or possibly a subset) of $\tau$-KMS states over $\mathfrak{A}$. These states, $\omega$, form a convex set with extremal points; a state is extremal in this set if, and only if, it is a factor state. On the other hand a state of such a system is factorial if, and only if, it possesses a strong spatial cluster property of the Powers type. We demonstrate that the states which are stable under "distant" dynamical perturbations are factor states and hence provide a connection with property $\mathrm{c}$.

The stability criterion is of the following nature. For each $P=P^{*} \in \mathfrak{A}$ we define local perturbations $\omega_{P}$ and $\tau^{P}$ of $\omega$ and $\tau$ and then consider sets of perturbations, typically perturbations which recede in configuration space, which leave the dynamics stable in the sense that:

$$
\left\|\tau_{t}^{P_{\alpha}}(A)-\tau_{t}(A)\right\| \underset{\alpha}{\longrightarrow} 0, \quad A \in \mathfrak{U} .
$$


The requirement of purity of $\omega$ is that

$$
\omega_{P_{\alpha}}(A) \underset{\alpha}{\longrightarrow} \omega(A), \quad A \in \mathfrak{U}
$$

and we show that this implies that $\omega$ is a factor state. Moreover we characterize exactly the set of stabilizers $\left\{P_{\alpha}\right\}$ necessary to enforce the equivalence of the factor property and this form of dynamical stability.

This stability criterion is possibly of greater interest for more complex models whose equilibrium states are no longer KMS with respect to a global dynamical group but satisfy this property with respect to a modular group which varies from state to state. In this context stability under distant perturbations again implies the factor property and hence spatial clustering. More generally this form of perturbation can isolate subphases of a general state and provide a basis for the decomposition of Gibbs states into pure phases.

\section{Boundary Perturbations}

Throughout this section we consider a pair $(\mathfrak{A}, \tau)$ consisting of $C^{*}$ algebra with identity, $\mathfrak{U}$, and a strongly continuous one parameter group $\tau$ of automorphisms of $\mathfrak{U}$. We associate to each self-adjoint $P \in \mathfrak{U}$ a perturbed group of automorphisms by the definition [1]

$$
\tau_{t}^{P}(A)=\tau_{t}(A)+\sum_{n \geqq 1} i^{n} \int_{0 \leqq s_{1} \leqq \ldots \leqq s_{n} \leqq t} d s_{1} \ldots d s_{n}\left[\tau_{s_{1}}(P),\left[\ldots\left[\tau_{s_{n}}(P), \tau_{t}(A)\right]\right]\right]
$$

and a similar formula for $t \leqq 0$. We will be particularly interested in sets of perturbations which leave $\tau$ asymptotically invariant.

Definition $1^{1}$. A stabilizer of $(\mathfrak{U}, \tau)$ is defined as a directed set $\mathscr{P}=\left\{P_{\alpha}\right\}$ of selfadjoint, $\tau$-analytic elements $P_{\alpha}$ of $\mathfrak{U}$ with the properties that $\left\|P_{\alpha}\right\|$ and $\left\|\tau_{i}\left(P_{\alpha}\right)\right\|$ are bounded uniformly $\alpha$ and

$$
\lim _{\alpha}\left\|\tau_{t}^{P_{\alpha}}(A)-\tau_{t}(A)\right\|=0, \quad A \in \mathfrak{U}, t \in \mathbb{R} .
$$

Next, let $\omega$ be a state over $\mathfrak{A}$ which satisfies the $\tau$-KMS condition [2]. For each perturbation $P=P^{*} \in \mathfrak{U}$ there exists a $\tau^{P}$-KMS vector state $\omega_{P}$ of $\omega$ given by $[3,4]$

where

$$
\omega_{P}(A)=\omega\left(W_{P} A\right) / \omega\left(W_{P}\right)=\omega\left(V_{P}^{*} A V_{P}\right) / \omega\left(V_{P}^{*} V_{P}\right)
$$

$$
W_{P}=1-\sum_{n \geqq 1}(-1)^{n} \int_{0 \leqq s_{1} \leqq} \int_{s_{n} \leqq 1} d s_{1} \ldots d s_{n} \tau_{i s_{n}}(P) \ldots \tau_{i s_{1}}(P)
$$

and

$$
V_{P}=1+\sum_{n \geqq 1}(-1)^{n} \int_{0 \leqq s_{1} \leqq \cdots \leqq s_{n} \leqq \frac{1}{2}} d s_{1} \ldots d s_{n} \tau_{i s_{1}}(P) \ldots \tau_{i s_{n}}(P) \text {. }
$$

In fact these formulas are only strictly valid for those $P$ such that $t \in \mathbb{R} \mapsto \tau_{t}(P) \in \mathfrak{A}$ has an analytic extension to the strip $0 \leqq 1 \mathrm{~m} t \leqq 1$. They can be extended to general $P$ by strong limiting in the representation space $\mathscr{H}_{\omega}$ associated with $\omega$ [3]. If $\pi_{\omega}$

1 The requirements of $\tau$-analyticity (see [2] and [7]) and uniform boundedness seem unnatural but are essentially justified by the characterization provided by Theorem 2 . 
and $\Omega_{\omega}$ denote the representation and cyclic vector associated with $\omega$ and $P_{1}, P_{2}$ are $\tau$-analytic one has the useful estimate [4]

$$
\left\|\pi_{\omega}\left(V_{P_{1}}\right) \Omega_{\omega}-\pi_{\omega}\left(V_{P_{2}}\right) \Omega_{\omega}\right\| \leqq \frac{\left\|P_{1}-P_{2}\right\|}{2} \exp \left\{\frac{\|P\|}{2}\right\}
$$

where $\|P\|=\operatorname{Max}\left(\left\|P_{1}\right\|,\left\|P_{2}\right\|\right)$. Another useful estimate [5] is the following

and hence

$$
\omega\left(W_{P}\right) \geqq e^{-\omega(P)} \geqq e^{-\|P\|}
$$

$$
\begin{aligned}
\left|\omega_{P_{1}}(A)-\omega_{P_{2}}(A)\right| & \leqq 2\|A\|\left\|\frac{\pi_{\omega}\left(V_{P_{1}}\right) \Omega_{\omega}}{\left\|\pi_{\omega}\left(V_{P_{1}}\right) \Omega_{\omega}\right\|}-\frac{\pi_{\omega}\left(V_{P_{2}}\right) \Omega_{\omega}}{\left\|\pi_{\omega}\left(V_{P_{2}}\right) \Omega_{\omega}\right\|}\right\| \\
& \leqq 2\|A\|\left\|P_{1}-P_{2}\right\| \exp \left\{\frac{3}{2}\|P\|\right\} .
\end{aligned}
$$

Our first result is the following

Theorem 1. Let $\omega$ be a $\tau$-KMS state over the $C^{*}$-algebra $\mathfrak{A}$. For each $P=P^{*} \in \mathfrak{A}$ let $\tau^{P}$ and $\omega_{P}$ denote the perturbed group of automorphisms and the perturbed $\tau^{P}$-KMS state, as defined above, respectively.

If $\omega$ is a factor state it follows that

$$
\lim _{\alpha} \omega_{P_{\alpha}}(A)=\omega(A), \quad A \in \mathfrak{A}
$$

for every stabilizer $\mathscr{P}=\left\{P_{\alpha}\right\}$ of $(\mathfrak{A}, \tau)$.

Proof. As $\left\|\tau_{i}\left(P_{\alpha}\right)\right\|$ is bounded uniformly in $\alpha$ it follows from the PhragmenLindelöf version of the maximum modulus principle (see [6] page 243) that $\left\|\tau_{i \gamma}\left(P_{\alpha}\right)\right\|$ is also uniformly bounded for $0 \leqq \operatorname{Im} \gamma \leqq 1$. One then concludes from (2) and (5) that $W_{P_{\alpha}} / \omega\left(W_{P_{\alpha}}\right)$ is uniformly bounded. Let $W$ be a weak limit point of this family represented on $\mathscr{H}_{\omega}$ and consider the state

$$
\hat{\omega}(A)=\left(\Omega_{\omega}, W \pi_{\omega}(A) \Omega_{\omega}\right), \quad A \in \mathfrak{A} .
$$

This state is a weak limit point of the $\omega_{P_{\alpha}}$ and by the following lemma is $\tau$-KMS.

Lemma 1. Let $\tau^{\alpha}$ be a directed set of strongly continuous one parameter groups of automorphisms of a $C^{*}$ algebra $\mathfrak{A}$ which converge strongly to $\tau$ i.e.

$$
\lim _{\alpha}\left\|\tau_{t}^{\alpha}(A)-\tau_{t}(A)\right\|=0, \quad A \in \mathfrak{U}, t \in \mathbb{R} .
$$

Further let $\omega_{\alpha}$ be a set of $\tau^{\alpha}$-KMS states over $\mathfrak{A}$ and $\omega$ a weak ${ }^{*}$ limit point of $\omega_{\alpha}$. It follows that $\omega$ is $\tau$-KMS.

Proof. To each $A \in \mathfrak{U}$ we associate a regularized $A_{\alpha}(f) \in \mathfrak{U}$ by the definition

$$
A_{\alpha}(f)=\int d t \tau_{t}^{\alpha}(A) f(t)
$$

where $f$ is a function whose fourier transform $\tilde{f}$ is $C^{\infty}$ with support in the interval $|p| \leqq a$. It follows by easy estimates that

where

$$
|f(t+i s)| \leqq b e^{a|s|} / \pi\left(1+t^{2}\right)
$$

$$
b=\operatorname{Max}\left(\|\tilde{f}\|_{1},\left\|\tilde{f}^{\prime \prime}\right\|_{1}\right) / 2 \text {. }
$$


Hence for any interval $I \subset \mathbb{R}$

$$
\begin{aligned}
& \left\|\tau_{z}(A(f))-\tau_{z}^{\alpha}\left(A_{\alpha}(f)\right)\right\| \\
& \leqq 2\|A\| \int_{s \notin I} d s|f(s-z)|+\int_{s \in I} d s|f(s-z)|\left\|\tau_{s}^{\alpha}(A)-\tau_{s}(A)\right\| \\
& \leqq \frac{2 b}{\pi} e^{a I m|z|}\left[\|A\| \int_{s \notin I-\operatorname{Re} z} d s \frac{1}{1+s^{2}}+\sup _{s \in I}\left\|\tau_{s}^{\alpha}(A)-\tau_{s}(A)\right\| \int_{s \in I-\operatorname{Rez} z} d s \frac{1}{1+s^{2}}\right] .
\end{aligned}
$$

Using the strong continuity of the $\tau^{\alpha}$ and their convergence on the real axis to $\tau$, we deduce that

$$
\lim _{\alpha}\left\|\tau_{z}^{\alpha}\left(A_{\alpha}(f)\right)-\tau_{z}(A(f))\right\|=0
$$

for all $z \in K$ where $K \subset \mathbb{C}$ is compact. Thus for all $A, B \in \mathfrak{A}$ one has

$$
\begin{aligned}
\omega(A(f) B) & =\lim _{\alpha} \omega_{\alpha}\left(A_{\alpha}(f) B\right) \\
& =\lim _{\alpha} \omega_{\alpha}\left(B \tau_{i}^{\alpha}\left(A_{\alpha}(f)\right)\right. \\
& =\omega\left(B \tau_{i}(A(f))\right)
\end{aligned}
$$

but this ensures that $\omega$ is $\tau$-KMS by the usual arguments involving analytic elements $[2,7]$.

Returning to the proof of Theorem 1 we deduce that

$$
\hat{\omega}(A)=\left(\Omega_{\omega}, W \pi_{\omega}(A) \Omega_{\omega}\right)
$$

is $\tau$-KMS with $W \in \pi_{\omega}^{\prime \prime}$. But it then follows from [8,9] (see also [10]) that $W \in \pi_{\omega}^{\prime \prime} \cap \pi_{\omega}^{\prime}$.

As $\omega$ is factorial this implies, however, that $W$ is the identity and $\hat{\omega}=\omega$. Thus we conclude that all weak limit points of the $W_{P_{\alpha}} / \omega\left(W_{P_{\alpha}}\right)$ are identical and the operators converge weakly. Hence the $\omega_{P_{\alpha}}$ converge in the weak* topology to $\omega$. This completes the proof of the theorem.

Next we wish to prove a converse to Theorem 1 but for this we need further structural assumptions on $\mathfrak{A}$ and $\omega$. The reason that more detailed structure is necessary is the possible non-existence of stabilisers of $(\mathfrak{H}, \tau)$. This does not affect the veracity of Theorem 1 but reduces it to an empty statement; it would, however, render the proof of the converse impossible.

We will consider algebras with the quasi-local structure encountered in quantum statistical mechanics and then restrict our attention to locally normal states. There are two possible choices of statistic, bose or fermi, of which we consider only the former. Our results can be extended to the fermi case with minor modifications (see, for example, [11]).

Specifically we define the $C^{*}$ algebra $\mathfrak{Q}$ to be a quasi-local algebra if it possesses a family $\mathfrak{A}_{\Lambda}$ of subalgebras, where $\Lambda$ runs over either the bounded open subsets of $\mathbb{R}^{v}$ or the finite subsets of $\mathbb{Z}^{v}$, satisfying the following;

1. $\bigcup \mathfrak{A}_{A}$ is norm dense in $\mathfrak{A}$.

2. $\Lambda_{1} \subset \Lambda_{2}$ implies $\mathfrak{U}_{\Lambda_{1}} \subseteq \mathfrak{A}_{\Lambda_{2}}$.

3. each $\mathfrak{A}_{A}$ is isomorphic to the algebra $\mathfrak{B}\left(\mathscr{H}_{A}\right)$ of all bounded operators on some Hilbert space $\mathscr{H}_{\Lambda}$. 
4. $\Lambda_{1} \cap \Lambda_{2}=\emptyset$ implies $\left[\mathfrak{A}_{\Lambda_{1}}, \mathfrak{A}_{\Lambda_{2}}\right]=0$.

A locally normal state over a quasi-local algebra $\mathfrak{A}$ is defined as a state $\omega$ with the property that

$$
\omega\left(\mathfrak{H}_{\Lambda}\right)=\operatorname{Tr}_{\mathscr{H}_{\Lambda}}\left(\varrho_{A} \mathfrak{H}_{\Lambda}\right)
$$

for each $\Lambda$, where $\varrho_{A}$ is a positive trace class operator. (The isomorphism between $\mathfrak{A}_{\Lambda}$ and $\mathfrak{B}\left(\mathscr{H}_{A}\right)$ is left implicit.)

Before proceeding we demonstrate that if $\mathfrak{A}$ is quasi-local, and $\tau$ arbitrary, then $(\mathfrak{A}, \tau)$ possesses stabilisers. We first remark that condition 4 above ensures the existence of a sequence $B_{n} \in \mathfrak{A}_{\Lambda+n}$ such that $\left\|B_{n}\right\|$ is uniformly bounded and

$$
\lim _{|n| \rightarrow \infty}\left\|\left[B_{n}, A\right]\right\|=0
$$

for all $A \in \mathfrak{A}$. Next form

$$
B_{n}(f)=\int d t \tau_{t}\left(B_{n}\right) f(t)
$$

where $f$ is a function whose Fourier transform is $C^{\infty}$ with compact support. It follows that $B_{n}(f)$ is $\tau$-analytic and $\left\|\tau_{i}\left(B_{n}(f)\right)\right\|$ is uniformly bounded in $n$,

$$
\left\|\tau_{i}\left(B_{n}(f)\right)\right\| \leqq\left\|B_{n}\right\| C_{f}
$$

where $C_{f}$ is independent of $n$ (compare the proof of Lemma 1). One also has for each interval $I \subset \mathbb{R}$

$$
\left\|\left[B_{n}(f), A\right]\right\| \leqq 2\left\|B_{n}\right\|\|A\| \int_{t \neq I} d t|f(t)|+\int_{t \in I} d t|f(t)|\left\|\left[B_{n}, \tau_{-t}(A)\right]\right\| .
$$

Exploiting the strong continuity of $\tau$, or using the Lebesque Lemma, one easily concludes that

for all $A \in \mathfrak{A}$.

$$
\lim _{n \rightarrow \infty}\left\|\left[B_{n}(f), A\right]\right\|=0
$$

Finally if $B_{n}$ is self-adjoint and $f$ real then $B_{n}(f)$ is self-adjoint and

$$
\begin{aligned}
\left\|\tau_{t}^{B_{n}(f)}(A)-\tau_{t}(A)\right\| & =\| \int_{0}^{t} d s \tau_{t_{n}^{B_{n}(f)}\left(\left[B_{n}(f), \tau_{s}(A)\right]\right) \|}^{t} \\
& \leqq \int_{0}^{t} d s\left\|\left[B_{n}(f), \tau_{s}(A)\right]\right\| .
\end{aligned}
$$

Combining these estimates and again using the Lebesque Lemma one has

$$
\lim _{n \rightarrow \infty}\left\|\tau_{t}^{B_{n}(f)}(A)-\tau_{t}(A)\right\|=0
$$

for all $A \in \mathfrak{A}$, i.e. $\left\{B_{n}(f)\right\}$ is a stabilizer of $(\mathfrak{A}, \tau)$.

We are now in a position to characterize the factorial $\tau$-KMS states of a quasilocal algebra. The following theorem restates a number of well-known facts, for completeness, but also gives a stability characterization.

Theorem 2. Let $\omega$ be a locally normal, $\tau$-KMS state, over a quasi-local algebra $\mathfrak{A}$. For each $P=P^{*} \in \mathfrak{A}$ let $\tau^{P}$ and $\omega_{P}$ denote the perturbed group of automorphisms and the perturbed $\tau^{P}$-KMS state respectively. 
The following conditions are equivalent

1. $\omega$ is a factor state.

2. Given $A \in \mathfrak{U}$ and $\varepsilon>0$ there is a $\Lambda$ such that

for all $B \in \bigcup_{\Lambda^{\prime} \cap \Lambda=\emptyset} \mathfrak{\Re}_{\Lambda^{1}}$.

$$
|\omega(A B)-\omega(A) \omega(B)|<\varepsilon\|B\|
$$

3. $\omega$ is extremal $\tau$-KMS.

4. For every stabiliser $\mathscr{P}=\left\{P_{\alpha}\right\}$ of $(\mathfrak{U}, \tau)$

$$
\lim _{\alpha} \omega_{P_{\alpha}}(A)=\omega(A), \quad A \in \mathfrak{U} .
$$

This theorem expresses the equivalence between the various characterizations of pure phases mentioned in the introduction. The equivalence between Conditions 1 and 2 is a property of the Powers type; proofs can be found in $[11,12]$. The equivalence of 1 and 3 is established in $[8,9]$ and summarized in [10]. The implication $1 \Rightarrow 4$ is established in Theorem 1 and it remains to prove that $4 \Rightarrow 1$.

It is the equivalence of Condition 4 with the other conditions that essentially justifies our definition of stabiliser. We will deduce $4 \Rightarrow 1$ by proving that if 1 is false then 4 is false. Assume that $\boldsymbol{Z}_{\omega}$ contains a non-trivial hermitian element $z$. For quasi-local algebras and locally normal states it is known [13,11] that

$$
3_{\omega}=\bigcap_{\Lambda}\left(\bigcup_{\Lambda^{\prime} \cap \Lambda=0} \pi_{\omega}\left(\mathfrak{U}_{\Lambda^{1}}\right)\right)^{\prime \prime} .
$$

This characterization, the Kaplansky density theorem, and the density of the $\mathfrak{U}_{\Lambda}$ in $\mathfrak{A}$, then ensure the choice of a directed set of self adjoint $B_{\alpha} \in \mathfrak{U}$ such that $\left\|B_{\alpha}\right\|$ is uniformly bounded

and

$$
\lim _{\alpha}\left\|\left[B_{\alpha}, A\right]\right\|=0, \quad A \in \mathfrak{A}
$$

$$
\lim _{\alpha}\left\|\left(\pi_{\omega}\left(B_{\alpha}\right)-z\right) \psi\right\|=0, \quad \psi \in \mathscr{H}_{\omega} .
$$

Forming the regularized $B_{\alpha}(f)$ as before we note that $\left\{B_{\alpha}(f)\right\}$ is a stabiliser of $(\mathfrak{U}, \tau)$.

Now as $\omega$ is $\tau$-KMS it is in particular $\tau$-invariant and there exists a strongly continuous one parameter group of unitaries $U_{\omega}$ acting on $\mathscr{H}_{\omega}$ such that

and

$$
\pi_{\omega}\left(\tau_{t}(A)\right)=U_{\omega}(t) \pi_{\omega}(A) U_{\omega}(t)^{-1}, \quad t \in \mathbb{R}, A \in \mathfrak{U}
$$

$$
U_{\omega}(t) z=z U_{\omega}(t), \quad t \in \mathbb{R}, z \in \mathcal{Z}_{\omega}
$$

(see for example [14]). Thus

$$
\begin{aligned}
& \left\|\left(\pi_{\omega}\left(B_{\alpha}(f)\right)-\int d t f(t) z\right) \psi\right\| \\
& \quad \leqq \int d t|f(t)|\left\|\left(\pi_{\omega}(B)-z\right) U_{\omega}(t) \psi\right\| .
\end{aligned}
$$

Taking $\int d t f(t)=1$ we deduce that $s-\lim _{\alpha} \pi_{\omega}\left(B_{\alpha}(f)\right)=z$. Now let us consider the convergence of the perturbed states associated with the stabilizer. These states are of the form

$$
\omega_{B_{\alpha}(f)}(A)=\left(\psi\left(B_{\alpha}(f)\right), \pi_{\omega}(A) \psi\left(B_{\alpha}(f)\right)\right) /\left\|\psi\left(B_{\alpha}(f)\right)\right\|^{2}
$$


where we have used Araki's notation [3]

$\psi(h)=\sum_{n \geqq 0}(-1)^{n} \int_{0 \leqq s_{1} \leqq \cdots \leqq s_{n} \leqq \frac{1}{2}} d s_{1} \ldots d s_{n} U_{\omega}\left(i s_{1}\right) h U_{\omega}\left(i\left(s_{2}-s_{1}\right)\right) \ldots U_{\omega}\left(i\left(s_{n}-s_{n-1}\right)\right) h \Omega_{\omega}$ for each $h \in \pi_{\omega}^{\prime \prime}$.

But Proposition 4.1. of [3] establishes that if $h_{\alpha} \in \pi_{\omega}^{\prime \prime}$ converges strongly to $h \in \pi_{\omega}^{\prime \prime}$ then $\psi\left(h_{\alpha}\right)$ converges strongly to $\psi(h)$. Thus $\psi\left(B_{\alpha}(f)\right)$ converges strongly to $\psi(z)$. But as $z$ commutes with $U_{\omega}$ the vector $\psi(z)$ is easily evaluated and one has

Therefore

$$
s-\lim _{\alpha} \psi\left(B_{\alpha}(f)\right)=e^{-z / 2} \Omega_{\omega} .
$$

$$
\lim _{\alpha} \omega_{B_{\alpha}(f)}(A)=\left(\Omega_{\omega} \pi_{\omega}(A) e^{-z} \Omega_{\omega}\right) /\left(\Omega_{\omega}, e^{-z} \Omega_{\omega}\right) \neq \omega(A)
$$

and Condition 4 is false. This proves the theorem. In fact we have established more concerning the structure of KMS states and stabilizers.

Theorem 3. Let $\omega$ be a locally normal $\tau$-KMS state over a quasi-local $C^{*}$ algebra $\mathfrak{A}$ and let $\hat{\omega}$ be any $\tau$-KMS state which is majorized by $\omega$.

Given $\varepsilon>0$ there exists a stabilizer $\mathscr{P}=\left\{P_{\alpha}\right\}$ of $(\mathfrak{A}, \tau)$ such that

$$
\sup _{A \in \mathscr{U}}\left|\lim _{\alpha} \omega_{P_{\alpha}}(A)-\hat{\omega}(A)\right| /\|A\|<\varepsilon .
$$

Proof. Each $\tau$-KMS state $\omega$ which is majorized by $\omega$ is of the form [8-10]

$$
\hat{\omega}(A)=\left(\Omega_{\omega}, T \pi_{\omega}(A) \Omega_{\omega}\right)
$$

where $T \in 3_{\omega}, 0 \leqq T \leqq 1$, and

$$
\left(\Omega_{\omega}, T \Omega_{\omega}\right)=1 .
$$

Now introduce $T_{n}$ by

$$
T_{n}=\left(T+\frac{1}{n}\right) /\left(1+\frac{1}{n}\right)
$$

and note that $0 \leqq T_{n} \leqq 1, T_{n} \in 3_{\omega}$, and

$$
\left(\Omega_{\omega}, T_{n} \Omega_{\omega}\right)=1 .
$$

The states $\omega_{n}$ determined by $T_{n}$, i.e.

$$
\omega_{n}(A)=\left(\Omega_{\omega}, T_{n} \pi_{\omega}(A) \Omega_{\omega}\right)
$$

are also $\tau$-KMS and satisfy the estimate

$$
\left|\omega_{n}(A)-\hat{\omega}(A)\right| \leqq 2\|A\| / n .
$$

Hence given $\varepsilon>0$ one can choose $n$ such that

$$
\sup _{A \in \mathscr{2}}\left|\omega_{n}(A)-\hat{\omega}(A)\right| /\|A\|<\varepsilon .
$$

Finally if $z_{n}$ is defined by $z_{n}=\log \left(1+\frac{1}{n}\right) /\left(T+\frac{1}{n}\right)$ one has $0 \leqq z_{n} \leqq \log n$ i.e. $z_{n}$ is bounded, and $e^{-z_{n}} / \omega\left(e^{-z_{n}}\right)=T_{n}$. 
But in the proof of Theorem 2 we have constructed a stabilizer such that each state of the form $\omega_{n}$ can be attained as a weak limit. This completes the proof of the theorem.

\section{Weak Boundary Perturbations}

In quantum statistical mechanics the basic assumptions used in the previous section are often invalid, e.g. there appears to be no pair $(\mathfrak{A}, \tau)$ with the assumed properties, associated with the free Bose gas. Thus in this section we attempt to draw some of the conclusions of the previous section under weaker circumstances.

Throughout this section $\mathscr{M}$ will denote a von Neumann algebra acting on a Hilbert space $\mathscr{H}$ and $U$ a strongly continuous one-parameter group of unitaries on $\mathscr{H}$ which implement automorphisms $\tau$ of $\mathscr{M}$, i.e.

$$
M \in \mathscr{M} \mapsto \tau_{t}(M)=U(t) M U(-t) \in \mathscr{M}
$$

for all $t \in \mathbb{R}, M \in \mathscr{M}$. Further $\omega$ will denote a state over $\mathscr{M}$ determined by a cyclic vector $\Omega \in \mathscr{H}$.

We will need a weaker notion of stabilizer

Definition 2. A stabilizer of $(\mathscr{M}, \tau, \omega)$ is defined as a directed set $\mathscr{P}=\left\{P_{\alpha}\right\}$ of self-adjoint $\tau$-analytic elements $P_{\alpha}$ of $\mathscr{M}$ with the properties that $\left\|P_{\alpha}\right\|$, and $\left\|\tau_{i}\left(P_{\alpha}\right)\right\|$, are bounded uniformly in $\alpha$ and

$$
\lim _{\alpha}\left\|\left(\tau_{t}^{P_{\alpha}}(M)-\tau_{t}(M)\right) \psi\right\|=0
$$

for all $M \in \mathscr{M}, t \in \mathbb{R}$ and $\psi \in \mathscr{H}$.

The first question that arises is whether stabilizers exist. We first demonstrate that $\left\{P_{\alpha}\right\}$ exist, for a general triplet $(\mathscr{M}, \tau, \omega)$ satisfying all the requirements except the $\tau$-analyticity and the boundedness of $\left\|\tau_{i}\left(P_{\alpha}\right)\right\|$. To construct such a sequence take $z \in \mathscr{M} \cap \mathscr{M}^{\prime}$ and choose $P_{\alpha} \in \mathscr{M}$ such that $P_{\alpha}$ converges strongly to $z$. Now if

and we define

$$
U(t)=e^{i H t}
$$

then one can show $[I]$ that

$$
U_{P_{\alpha}}(t)=e^{i\left(H+P_{\alpha}\right) t}
$$

$$
\tau_{t}^{P_{\alpha}}(M)=U_{P_{\alpha}}(t) M U_{P_{\alpha}}(-t) .
$$

Thus one estimates, as in Section 2, that

$$
\begin{aligned}
\left\|\left(\tau_{t}^{P_{\alpha}}(M)-\tau_{t}(M)\right) \psi\right\| \leqq & \int_{0}^{|t|} d s\left\|\left[P_{\alpha}, \tau_{s}(M)\right] U_{P_{\alpha}}(s-t) \psi\right\| \\
\leqq & 2\left\|P_{\alpha}\right\|\|M\| \int_{0}^{|t|} d s\left\|\left(U_{P_{\alpha}}(s)-U_{z}(s)\right) \psi\right\| \\
& +\int_{0}^{|t|} d s\left\|\left[P_{\alpha}-z, \tau_{s}(M)\right] U_{z}(s-t) \psi\right\| .
\end{aligned}
$$

Hence invoking the uniform boundedness principle and the Lebesque Lemma we deduce that $\tau_{t}^{P_{\alpha}}(M)$ converges strongly to $\tau_{t}(M)$ whenever $U_{P_{\alpha}}(t)$ converges strongly to $U_{z}(t)$. But

$$
\left\|\left(U_{P_{\alpha}}(t)-U_{z}(t)\right) \psi\right\| \leqq \int_{0}^{|t|} d s\left\|\left(P_{\alpha}-z\right) U_{z}(s) \psi\right\|
$$

and hence the convergence is ensured. 
If the triplet $(M, \tau, \omega)$ also satisfies the requirement that the centre of $\mathscr{M}$ is left pointwise invariant under $\tau$, i.e. each $z \in \mathscr{M} \cap \mathscr{M}^{\prime}$ commutes with $U(t)$ for all $t \in \mathbb{R}$, then we can regularize the above set $\left\{P_{\alpha}\right\}$ with a regularization function $f$, as in Section 2, and deduce that the regularized set $\left\{P_{\alpha}(f)\right\}$ satisfy all the requirements of Lemma 2. The argument proceeds as above but uses the strong convergence of $P_{\alpha}(f)$ to $z$ times the integral of $f$.

Theorem 4. Consider a triplet $(\mathscr{M}, \tau, \omega)$ as defined above and assume that $\omega$ is a $\tau$-KMS state over $\mathscr{M}$.

If $\omega$ is stable under perturbations in the sense that

$$
\lim _{\alpha} \omega_{P_{\alpha}}(M)=\omega(M), \quad M \in \mathscr{M}
$$

for all stabilizers $\mathscr{P}=\left\{P_{\alpha}\right\}$ of $(\mathscr{M}, \tau, \omega)$ then it follows that $\mathscr{M}$ is a factor.

If, on the other hand, $\hat{\omega}$ is a $\tau$-KMS state majorized by $\omega$ then, for each $\varepsilon>0$, there exists a stabilizer of $(\mathscr{M}, \tau, \omega)$ such that

$$
\sup _{M \in \mathscr{M}}\left|\hat{\omega}(M)-\lim _{\alpha} \omega_{P_{\alpha}}(M)\right| /\|M\|<\varepsilon .
$$

The proof of the theorem copies the proofs of Theorem 2 and 3. One also uses the new definition of a stabilizer, the pointwise invariance of $\mathscr{M} \cap \mathscr{M}^{\prime}$ under $\tau$ which follows from the KMS condition (see, for example $[10,14]$ ) and the fact established above, that each $z \in \mathscr{M} \cap \mathscr{M}^{\prime}$ can be strongly approximated by a stabilizer.

Acknowledgement. One of us (HN) is grateful to l'Institut Français de Vienne who enabled this collaboration and the CNRS, Marseille, for its hospitality.

\section{References}

1. Robinson, D. W.: Commun. math. Phys. 31, 171 (1973); see also Streater, R.F.: Commun. math. Phys. 7, 93 (1968)

2. Haag, R., Hugenholtz, N., Winnink, M.: Commun. math. Phys. 12, 215 (1967)

3. Araki,H.: Pub. Res. Inst. Math. Sci. Kyoto, 9, 87 (1973)

4. Robinson, D. W.: Proc. Varenna Summer School (1973)

5. Araki,H.: Golden-Thompson and Peierls-Bogoliubov inequalities. Kyoto (preprint)

6. Rudin, W.: Real and complex analysis. London-New York: 1970 McGraw-Hill

7. Kastler, D., Pool, J. C. T., Poulsen, E. T.: Commun. math. Phys. 12, 175 (1969)

8. Araki, H.: Commun. math. Phys. 14, 120 (1969)

9. Lanford, O.E.: Systèmes à un nombre infini de degrés de liberté. CNRS, Paris (1969)

10. Ruelle,D.: Cargèse lectures in physics. New York: Gordon and Breach 1970

11. Robinson, D. W.: Commun. math. Phys. 41, 79-88 (1975)

12. Haag, R., Kadison, R., Kastler, D.: Commun. math. Phys. 16, 81 (1970)

13. Ruelle, D.: J. Funct. Ann. 6, 110 (1970)

14. Winnink, M.: Cargèse lectures in physics. New York: Gordon and Breach 1970

Communicated by K. Hepp and J. L. Lebowitz

Heidi Narnhofer

Institut für Theoretische Physik

der Universität Wien

Boltzmangasse 5

A-1090 Wien, Austria
Derek W. Robinson

Centre de Physique Théorique

C. N.R.S.

31, chemin J. Aiguier

F-13274 Marseille Cedex 2, France 
